\title{
Aller de l'avant dans la lutte contre la tuberculose
}

\author{
Taylor G. ${ }^{1^{*}}$ \\ ${ }^{1}$ Sous-administrateur en chef de la santé publique, Agence de la santé publique du Canada, Ottawa (Ontario) \\ ${ }^{*}$ Auteur-ressource : Gregory.Taylor@phac-aspc.gc.ca
}

Le 24 mars est la Journée mondiale de lutte contre la tuberculose. Cette date sert à réfléchir à la situation actuelle de la tuberculose au Canada et à la façon dont nous contribuons aux efforts d'éradication de cette maladie à l'échelle mondiale.

La bonne nouvelle est que le Canada présente l'un des plus faibles taux de tuberculose au monde. Par contre, certaines sous-populations continuent d'être touchées de façon disproportionnée. Les Autochtones et les personnes nées à l'étranger ayant immigré au Canada depuis des pays où la tuberculose est très répandue ont plus de risques de contracter la maladie que les personnes non autochtones nées au Canada. Les autres souspopulations vulnérables sont les sans-abri, les détenus sous responsabilité fédérale et les personnes vivant avec le virus de l'immunodéficience humaine $(\mathrm{VIH})$. Pour un résumé des tendances de la tuberculose au Canada, voir le prochain article intitulé La tuberculose au Canada : 1924-2012 (1).

Dans l'ensemble, les dernières nouvelles concernant la tuberculose ne prêtent guère à l'optimisme. La tuberculose reste l'une des maladies infectieuses les plus répandues au monde. Chaque année, elle infecte près de neuf millions de personnes et cause plus de un million de décès (2). À l'échelle planétaire, la tuberculose est la principale cause de décès chez les personnes vivant avec le VIH. Pour une maladie qui peut être évitée et guérie, cela est pour le moins déconcertant.

Le Partenariat Halte à la tuberculose, sous l'égide de l'Organisation mondiale de la Santé (OMS), travaille avec des experts en la matière et des intervenants du monde entier sur le Plan mondial Halte à la tuberculose 2006 2015 (2). Le plan a défini que nous disposions, au niveau mondial, des éléments nécessaires pour stopper l'épidémie de tuberculose. Les stratégies du plan comprennent la participation des fournisseurs de soins, l'autonomisation des personnes atteintes de tuberculose, le renforcement des systèmes de santé et la mise à disposition de traitements de qualité, en particulier pour les cas difficiles présentant une co-infection avec le VIH et une pharmacorésistance multiple.

Ces dernières années, on s'est rapproché des objectifs mondiaux de réduction des cas de tuberculose; l'incidence de la maladie a commencé à décliner et la mortalité due à la tuberculose est en baisse (3). Le Partenariat Halte à la tuberculose demande à tous les États membres de l'OMS de continuer d'orienter leurs efforts en fonction de ce plan. C'est avec fierté que je souligne que le Canada contribue au succès du Plan mondial. Par l'intermédiaire du Conseil du Réseau pancanadien de santé publique (RSP), les gouvernements fédéral, provinciaux et territoriaux ont défini 12 éléments essentiels des programmes de prévention et de contrôle de la tuberculose. En 2012, le RSP a publié les Orientations pour les programmes de prévention et de contrôle de la tuberculose au Canada. Le document d'orientation cerne les besoins du système de santé en matière de détection précoce, de signalement et de traitement, mais également les besoins en matière de formation des professionnels, de sensibilisation communautaire et de meilleures pratiques pour composer avec les déterminants de la santé, sociaux ou autres. Pour plus de renseignements, vous trouverez dans ce numéro un résumé de ce document (4). Depuis que ce travail a été effectué, la Colombie-Britannique (5) et la Saskatchewan (6) ont publié leurs propres stratégies de prévention et de contrôle de la tuberculose qui cadrent étroitement avec les meilleures pratiques décrites par le RSP. L'Agence de la santé publique du Canada (l'Agence) salue ces initiatives et invite d'autres provinces/territoires à mettre sur pied leurs propres approches axées sur la population.

L'Agence, en partenariat avec la Société canadienne de thoracologie, a récemment publié la $7^{\mathrm{e}}$ édition des Normes canadiennes pour la lutte antituberculeuse, qui a été révisée en profondeur. Ce numéro présente un résumé des modifications (7), qui comprennent de nouvelles approches diagnostiques pour les infections tuberculeuses actives et latentes, des changements radicaux concernant les recommandations relatives au 
traitement de la tuberculose active, latente et pharmacorésistante, ainsi que des modifications considérables des recommandations en matière de traitement en cas de co-infection par le VIH. Le suivi des contacts et la gestion des éclosions sont traités différemment, et la nouvelle édition comprend également un chapitre sur l'adaptation des programmes sur le plan culturel.

En outre, le Canada a pris part aux efforts de contrôle de la tuberculose au-delà de ses frontières. Le Fonds pour l'extension des services de lutte antituberculeuse, financé grâce à une subvention de 120 millions de dollars du gouvernement du Canada, est un parfait exemple du rôle fondamental de partenariat que joue notre pays pour repérer et traiter les personnes atteintes de tuberculose dans certains des pays les plus pauvres de la planète. Le Canada contribue également aux efforts internationaux par une recherche novatrice. De nouvelles méthodes d'enquête, combinées à une transition vers des modèles de prévention axés sur la mobilisation communautaire plutôt que sur le comportement individuel, montrent des résultats prometteurs (8-11). De tels projets pilotes pourraient s'appliquer à la fois au Canada et dans le monde entier.

En dépit de toutes ces initiatives, il reste encore beaucoup à faire au Canada. On a toujours cruellement besoin de la participation ininterrompue des fournisseurs de soins et de l'autonomisation des personnes atteintes de tuberculose. Nous devons continuer à travailler ensemble à l'échelle locale et nationale ainsi qu'en collaboration avec nos partenaires internationaux pour traiter cet enjeu fondamental. Les partenariats élaborés par l'intermédiaire du RSP et d'autres organismes fédéraux, provinciaux et territoriaux nous permettront d'avancer vers notre objectif en tant que pays et en tant que défenseur du progrès de la santé mondiale.

\section{Références}

(1) Gallant V., Ogunnaike-Cooke S. et McGuire M. La tuberculose au Canada : 1924-2012. RMTC 2014;6:107-117.

(2) Partenariat Halte à la tuberculose et Organisation mondiale de la Santé. Plan mondial Halte à la tuberculose 2006-2015. Genève, Organisation mondiale de la Santé, 2006 (WHO/HTM/STB/2006.35). Accès : http://www.stoptb.org/assets/documents/global/plan/StopTB_GlobalPlan_FR_web.pdf

(3) Organisation mondiale de la Santé. Journée mondiale de lutte contre la tuberculose, 24 mars 2013. Genève. Accès : http://www.who.int/campaigns/tb-day/2013/fr/

(4) Lord L., au nom du Conseil du Réseau pancanadien de santé publique. Résumé des Orientations pour les programmes de prévention et de contrôle de la tuberculose au Canada du Conseil du Réseau pancanadien de santé publique. RMTC 2014;6:118-121.

(5) BC Communicable Disease Policy Advisory Committee. BC Strategic Plan for Tuberculosis Prevention, Treatment and Control. 2012. Accès : http://www.bccdc.ca/NR/rdonlyres/371821DC-D135-4BC6-8AD94F09CF667B29/0/BC_Strategic_Plan_Tuberculosis.pdf

(6) TB Partnership Working Group. Saskatchewan Provincial Tuberculosis Strategy 2013-2018: Prevention, Treatment, Education and Control. 2013. Accès : http://www.health.gov.sk.ca/tb-strategy-pdf

(7) Gale-Rowe M., Menzies R., Sutherland J. et Wong T. au nom des éditeurs et auteurs. Points saillants de la $7^{\mathrm{e}}$ édition des Normes canadiennes pour la lutte antituberculeuse. RMTC 2014;6:122-127.

(8) Cadre de travail national sur la santé pulmonaire. Projets - Phase II : Taima TB et Sensibilisation à la tuberculose des jeunes autochtones et non autochtones au Canada : un programme éducatif de santé publique historique et socio-culturel. Accès : http://www.cadretravailpulmonaire.ca/investissementf\%C3\%A9d\%C3\%A9ral/projets-phase-ii

(9) Cook V., Shah L. et Gardy J. Modern contact investigation methods for enhancing tuberculosis control in Aboriginal communities. Int J Circumpolar Health 2012;71:18643. http://dx.doi.org/10.3402/ijch.v7i0.18643 
(10) Jensen M., Lau A., Langlois-Klassen D., Boffa J., Manfreda J. et Long R. A population-based study of tuberculosis epidemiology and innovative service delivery in Canada. Int J Tuberc Lung Dis 2012;16(1):43-49. Accès : http://www.ncbi.nlm.nih.gov/pubmed/?term=A+populationbased+study+of+tuberculosis+epidemiology+and+innovative+service+delivery+in+Canada

(11) Orr P. Adherence to tuberculosis care in Canadian Aboriginal populations, Part 2: a comprehensive approach to fostering adherent behaviour. Int J Circumpolar Health 2012;70(2):128-140. Accès : http://www.ncbi.nlm.nih.gov/pubmed/?term=Adherence+to+tuberculosis+care+in+Canadian+populations+ Part+2\%3A+a+comprehensive+approach+to+fostering+adherent+behaviour 